\title{
CORRECTION
}

\section{Correction to: Special issue on recommender system}

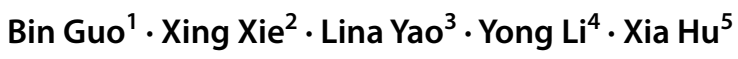

Published online: 9 January 2020

(c) China Computer Federation (CCF) 2020

\section{Correction to: \\ CCF Transactions on Pervasive Computing and Interaction (2019) 1:237-239 \\ https://doi.org/10.1007/s42486-019-00024-z}

On the title page, author Prof. Cecilia Mascolo should be removed. The mistake was caused by using an earlier unconfirmed version of guest editors for this special issue.

The original article can be found online at https://doi.org/10.1007/ s42486-019-00024-z.

\section{Bin Guo}

guob@nwpu.edu.cn

Xing Xie

xie.xing@microsoft.com

Lina Yao

lina.yao@unsw.edu.au

Yong $\mathrm{Li}$

liyong07@tsinghua.edu.cn

$\mathrm{Xia} \mathrm{Hu}$

hu@cse.tamu.edu

1 Northwestern Polytechnical University, Xian, China

2 Microsoft Research Asia, Bejing, China

3 The University of New South Wales, Sydney, Australia

4 Tsinghua University, Bejing, China

5 Texas A and M University, College Station, USA 